\title{
Seletividade de produtos alternativos a Telenomus podisi Ashmead (Hymenoptera: Scelionidae)
}

\section{Selectivity of alternative products to Telenomus podisi Ashmead (Hymenoptera: Scelionidae)}

\author{
Lisonéia Fiorentini Smaniotto ${ }^{1}$; Alfredo de Gouvea ${ }^{2}$; Michele Potrich ${ }^{2 *}$; \\ Everton Ricardi Lozano da Silva²; Jovenil da Silva ${ }^{3}$; Carla Samanta Pegorini ${ }^{4}$
}

\begin{abstract}
Resumo
O objetivo deste trabalho foi avaliar o efeito de produtos alternativos sobre o parasitoide de ovos Telenomus podisi (Ashmead) (Hymenoptera: Scelionidae). Os produtos utilizados foram Óleo de Gerânio, Natualho ${ }^{\circledR}$, Pironat ${ }^{\circledR}$, Rotenat $^{\circledR}$, Natuneem $^{\circledR}$ e Compostonat, na concentração recomendada pelo fabricante. Foram pulverizados 0,2 mL das caldas sobre cartelas contendo 25 ovos de Euschistus heros (Fabricius) (Hemiptera: Pentatomidae) e, como testemunha água destilada esterilizada. Para o teste com chance de escolha, uma fêmea de T. podisi foi confinada em tubo de vidro, com duas cartelas contendo ovos de E. heros, sendo uma pulverizada com o tratamento e a outra com água destilada esterilizada. No teste sem chance de escolha, uma fêmea de T. podisi foi confinada em um tubo de vidro contendo uma cartela de ovos de E. heros, por um período de $24 \mathrm{~h}$, sendo a cartela pulverizada com o produto previamente ou posteriormente ao parasitismo. Em ambos os experimentos avaliou-se a porcentagem de parasitismo, porcentagem de emergência de adulto dos parasitoides, período ovo-adulto e razão sexual. No teste com chance de escolha, os produtos Compostonat, Natualho ${ }^{\circledR}$ e Óleo de Gerânio reduziram significativamente a taxa de parasitismo. Redução semelhante também foi observada no teste sem chance de escolha para Natualho ${ }^{\circledR}$ e Óleo de Gerânio no pré e pós-parasitismo. No pré-parasitismo Compostonat e Óleo de Gerânio reduziram a emergência e, o período ovo-adulto foi afetado por Natualho ${ }^{\circledR}$. No pósparasitismo verificou-se redução no número de ovos com sinal de parasitismo quando pulverizado o produto Rotenat ${ }^{\mathbb{}}$. A razão sexual dos adultos emergidos foi reduzida por Natualho ${ }^{\circledR}$ e Óleo de Gerânio. Natuneem ${ }^{\circledR}$ foi o único produto seletivo a T. podisi em todos os parâmetros avaliados.

Palavras-chave: Euschistus heros, organismos não-alvos, parasitoides
\end{abstract}

\begin{abstract}
The objective of this study was to evaluate the selectivity of alternative products to the egg parasitoid Telenomus podisi (Ashmead) (Hymenoptera: Scelionidae). The products used were Oil Geranium, Natualho $^{\circledR}$, Pironat ${ }^{\circledR}$, Rotenat ${ }^{\mathbb{}}$, Natuneem ${ }^{\circledR}$ and Compostonat at the concentration recommended by manufacturer. Was sprayed $0.2 \mathrm{~mL}^{-1}$ of grout on cards containing 25 eggs of Euschistus heros (Fabricius) (Hemiptera: Pentatomidae) and sterile distilled water as a control. For the free-choice test, a female $T$.
\end{abstract}

${ }^{1}$ M.e em Agronomia, Universidade Tecnológica Federal do Paraná, UTFPR, Campus Pato Branco, Pato Branco, PR. E-mail: liso_smaniotto@hotmail.com

2 Profs. Drs. da UTFPR, Campus Dois Vizinhos, Dois Vizinhos, PR. E-mail: alfredo@utfpr.edu.br; michelepotrich@utfpr.edu.br; profmichele@gmail.com; evertonlricardi@utfpr.edu.br

3 M.e em Agronomia. Empresa Brasileira de Pesquisa Agropecuária, Embrapa Soja, Rod. Carlos João Strass, Distrito de Warta. Londrina, PR. E-mail: nil-bio@hotmail.com

${ }^{4}$ Graduanda em Zootecnista, Bolsista de Iniciação Científica CNPq. UTFPR, Campus Dois Vizinhos, PR. E-mail: carla_samantar@ hotmail.com

* Autor para correspondência 
podisi was confined in a glass tube with two cards containing eggs of $E$. heros one being sprayed with treatment and other with sterile distilled water. In the no-choice test, a female of $T$. podis $i$ was confined in a glass tube containing a card with eggs of $E$. heros, for a period of $24 \mathrm{~h}$, the card being sprayed with the product prior or subsequent to parasitism. The parasitism percentage, emergence percentage, eggadult period and sex ratio were evaluated. In free-choice test, the products Compostonat, Natualho ${ }^{\circledR}$ and Geranium Oil reduced significantly the percentage of parasitism. Similar reduction was also observed in no-choice test for Natualho ${ }^{\circledR}$ and Geranium oil in pre and post-parasitism. In pre-parasitism Compostonat and Geranium oil reduced the emergence and egg-adult period was affected by Natualho ${ }^{\circledR}$. In post- parasitism there was a reduction in the number of eggs with signs of parasitism when the product sprayed was Rotenat ${ }^{\circledR}$. The sex ratio of emerged adults was reduced by Natualho ${ }^{\circledR}$ and Geranium Oil. Natuneem ${ }^{\circledR}$ was the only product selective to T. podisi in all parameters evaluated.

Key words: Euschistus heros, non-target organisms, parasitoids

\section{Introdução}

Euschistus heros (Fabricius) (Hemiptera: Pentatomidae), também conhecido como percevejo marrom, já foi considerado uma praga secundária na cultura da soja, porém, nos últimos anos tem se destacado como uma das principais pragas desta cultura, com incidências nas regiões Sul e Centro Oeste do Brasil (MEDEIROS; MEGIER, 2009; GODOY et al., 2010).

E. heros se alimenta principalmente da soja, provocando danos durante o desenvolvimento da semente. Na fase inicial o ataque resulta no aborto das sementes, enquanto na fase de enchimento e maturação da vagem pode ocasionar deformações nas sementes. A atividade alimentar do inseto também atrasa o desenvolvimento e a maturação foliar, além de possibilitar a entrada de micro-organismos nas sementes (SCHAEFER; PANIZZI, 2000), que podem causar manchas de coloração escura e/ou esbranquiçadas, alterações na composição química e redução do poder germinativo (NUNES; CORRÊAFERREIRA, 2002; BELORTE et al., 2003).

Para o controle de $E$. heros são indicados cerca de 26 produtos dos grupos organofosforados, trizaois, neonicotinoides, piretroides, ciclodienoclorados, metilcarbamato de benzofuranila (AGROFIT, 2012). Além do controle químico, o controle biológico utilizando parasitoides de ovos, como Telenomus podisi (Ashmead) (Hymenoptera: Scelionidae), pode ser uma alternativa viável. Este inseto é capaz de parasitar ovos de E. heros e também de Piezodorus guildinii Westwood (Hemiptera: Pentatomidae) e Nezara viridula Linnaeus (Hemiptera: Pentatomidae) (CORRÊAFERREIRA; MOSCARDI, 1995; PACHECO; CORRÊA-FERREIRA, 2000).

Além dos parasitoides de ovos, a utilização de produtos conhecidos como "alternativos" vem se destacando na cultura da soja, em especial em cultivos orgânicos (PERES; CORRÊA-FERREIRA, 2006; CORRÊA-FERREIRA; PERES, 2006). Esses produtos são utilizados de forma isolada ou concomitantemente a outros métodos de controle, entretanto, podem ser prejudiciais a organismos não alvos.

Em estudo referente ao efeito de extratos de meliáceas sobre o parasitismo de Trichogramma pretiosum Riley (Hymenoptera: Trichogrammatidae), o extrato aquoso de sementes de nim (Azadirachta indica A. Juss) (10\%) reduziu significativamente o número de ovos de Anagasta kuehniela (Zeller) (Lepidoptera: Pyralidae) parasitados e a emergência de adultos, enquanto o extrato de folhas de Trichilia pallida Swartz não afetou estas variáveis (GONÇALVESGERVÁSIO; VENDRAMIM, 2004). O óleo emulsionável de nim também reduziu o parasitismo de Trichogramma galloi Zucchi (Hymenoptera: Trichogrammatidae) em ovos de Diatraea saccharalis (Fabricius) (Lepidoptera: Crambidae) (BROGLIO-MICHELETTI; SANTOS; PEREIRA-BARROS, 2006). Em avaliação de diversos produtos alternativos sobre $T$. pretiosum, 
Silva (2010) verificou que apenas o extrato de crisântemo Chrysanthemum cinerariaefolium Trev. (Asteraceae) e a calda sufocálcica reduziram o número de ovos de $A$. kuehniella parasitados.

Embora os produtos alternativos possam apresentar menor toxicidade quando comparados aos sintéticos, também podem afetar direta ou indiretamente parâmetros biológicos de parasitoides, como o parasitismo, a emergência, o período ovoadulto, a longevidade e a razão sexual. Contudo, as informações sobre os efeitos dessas interações são escassas, sendo necessários estudos específicos de seletividade de produtos utilizados na cultura da soja ou próximos a ela, que possam contaminar refúgios naturais dos parasitoides ou que, por deriva, possam atingir a cultura. Assim, o objetivo deste trabalho foi avaliar a seletividade de produtos alternativos a T. podisi.

\section{Material e Métodos}

\section{Criação e manutenção de T. podisi e E. heros}

Foram utilizados ovos de E. heros e fêmeas de T. podisi, obtidos no Laboratório de Bioecologia e Criação de Percevejos da Empresa Brasileira de Pesquisa Agropecuária (EMBRAPA - Soja). As fêmeas do parasitoide, com até $72 \mathrm{~h}$ de emergência (utilizadas com esta idade em todos os bioensaios), foram colocadas em tubos de vidro de fundo chato, juntamente com cartelas com ovos de E. heros. Os tubos foram acondicionados em câmara climatizada (26 $\pm 2{ }^{\circ} \mathrm{C}$, UR de $75 \pm 10 \%$, fotofase de $12 \mathrm{~h}$ ). Após $24 \mathrm{~h}$, as fêmeas foram retiradas e os tubos mantidos nas mesmas condições até a emergência dos adultos, os quais foram transferidos para frascos de criação (pet adaptado com $12 \mathrm{~cm}$ de diâmetro $\times 15 \mathrm{~cm}$ de altura, contendo algodão hidrofílico ao fundo) e alimentados com um filete de mel orgânico, depositado com um pincel fino na lateral dos frascos. Os insetos adultos permaneceram nos frascos até a utilização nos experimentos, por um tempo máximo de 72 horas.

\section{Bioensaios de seletividade}

Para os bioensaios de seletividade foram realizados os testes com chance de escolha e sem chance de escolha, sendo que, para este último, foram utilizadas duas estratégias de aplicação dos produtos: pré e pós-parasitismo. Foram utilizados produtos certificados para uso em diferentes culturas, com composição e concentração recomendada descritas na Tabela 1. Estes produtos foram obtidos em lojas especializadas, sendo estes certificados para uso em diferentes culturas (Tabela 1). As caldas dos produtos foram preparadas na concentração recomendada pelo fabricante, em frascos contendo $100 \mathrm{~mL}$ de água destilada esterilizada. Para o processo de pulverização, foram preparadas cartelas de 1,0 $\times 5,0 \mathrm{~cm}$, contendo 25 ovos de E. heros não parasitados, arranjadas aos pares sobre uma bancada e, com um aerógrafo Pneumatic Sagyma ${ }^{\circledR}$ acoplado a um compressor de ar, com pressão constante de 1,2 Kgf. $\mathrm{cm}^{-1}$, foram pulverizadas com $0,2 \mathrm{~mL}$ da calda do produto, na concentração recomendada pelo fabricante, volume suficiente para cobrir os ovos. Na testemunha, as cartelas com ovos foram pulverizadas com água destilada esterilizada. 
Tabela 1. Produtos alternativos empregados em cultivos orgânicos e utilizados nos experimentos, composição e concentração recomendada.

\begin{tabular}{lll}
\hline \multicolumn{1}{c}{ Produto } & \multicolumn{1}{c}{ Composição $^{1}$} & \multicolumn{1}{c}{$\begin{array}{c}\text { Concentração } \\
\text { recomendada }\end{array}$} \\
\hline Compostonat & Nim, timbó, gerânio, pimenta longa e outros extratos & $100 \mathrm{~mL} / 100 \mathrm{~L}$ \\
Natualho $^{\circledR}$ & Extrato de alho & $30 \mathrm{~mL} / 100 \mathrm{~L}$ \\
Natuneem $^{\circledR}$ & Óleo de nim, revigorante orgânico, óleo de mamona, extratos & $50 \mathrm{~mL} / 100 \mathrm{~L}$ \\
Óleo de Gerânio $_{\text {Pironat }^{\circledR}}$ & vegetais bioativos e veículo & $100 \mathrm{~mL} / 100 \mathrm{~L}$ \\
Rotenat $^{\circledR}$ & Linalol, Citronelol e Geraniol & $100 \mathrm{~m} \mathrm{L/100} \mathrm{L}$ \\
\hline
\end{tabular}

${ }^{1}$ Composição informada pelo fabricante.

Fonte: Elaboração dos autores.

\section{Teste com chance de escolha}

Para cada tratamento foram pulverizadas 20 cartelas com a calda e 20 cartelas com água destilada esterilizada. Estas foram deixadas por 5 minutos em capela de fluxo laminar para a evaporação do excesso de água. Em seguida, uma cartela pulverizada com a calda e outra pulverizada com a água foram acondicionadas em um tubo de vidro de fundo chato $(10 \mathrm{~cm}$ comprimento $\times 2,5$ $\mathrm{cm}$ de diâmetro), juntamente com uma fêmea de T. podisi. Os tubos foram fechados com filme de PVC e mantidos em câmara climatizada $\left(26 \pm 2{ }^{\circ} \mathrm{C}\right.$; UR de $75 \pm 10 \%$; fotofase de $12 \mathrm{~h}$ ). Após $24 \mathrm{~h}$ as fêmeas foram retiradas e os tubos permaneceram nas mesmas condições. Ao quinto dia avaliou-se a quantidade de ovos parasitados, identificados pela coloração enegrecida, sinal característico do parasitismo.

A taxa de parasitismo foi calculada comparando cada um dos produtos alternativos com a respectiva testemunha, sendo o número de ovos parasitados em ambos (testemunha e tratamento), considerado como $100 \%$, conforme a equação: Taxa Ptrat $=$ [OpTrat/(OpTrat + OPTest) $\times 100]$, onde: TaxaPtrat $=$ taxa de parasitismo no tratamento, OpTrat $=$ ovos parasitados no tratamento (inseticidas alternativos) e OPTest $=$ ovos parasitados na testemunha. Para a obtenção da taxa de parasitismo da testemunha, subtraiu-se a taxa de parasitismo do tratamento de 100\% (POTRICH, 2010). Os dados foram analisados pelo teste não paramétrico de Wilcoxon, com auxílio do programa estatístico BioEstat 5.0 (AYRES et al., 2007).

\section{Teste sem chance de escolha}

Pulverização pré-parasitismo: Para cada tratamento e para a testemunha foram preparadas 20 cartelas (repetições) com ovos de E. heros não parasitados. A metodologia de pulverização, a secagem das cartelas e as condições de temperatura, umidade relativa e fotofase foram as mesmas descritas no experimento anterior. Cada cartela foi colocada em um tubo de vidro de fundo chato, juntamente com uma fêmea de T. podisi, conforme metodologia adaptada de Potrich et al. (2009). Após 24 h, as fêmeas foram retiradas e os ovos foram observados diariamente até a emergência dos parasitoides, no qual se avaliou: porcentagem de parasitismo, porcentagem de emergência, período ovo-adulto de machos e de fêmeas e razão sexual dos adultos emergidos.

Pulverização pós-parasitismo: Vinte cartelas com ovos não parasitados de E. heros foram alocadas individualmente em tubos de vidro de fundo chato, juntamente uma fêmea de T. podisi. Os tubos foram fechados com filme de PVC e acondicionados em câmara climatizada nas mesmas condições descritas anteriormente. Após $24 \mathrm{~h}$ as fêmeas foram retiradas e as cartelas foram pulverizadas com um dos 
produtos alternativos (calda). As cartelas foram, novamente, individualizadas, acondicionadas nos tubos e mantidas nas mesmas condições em câmara climatizada. Os mesmos procedimentos foram realizados para a testemunha. As variáveis avaliadas foram as mesmas descritas para o teste com pulverização pré-parasitismo.

Para ambos os bioensaios o delineamento experimental foi inteiramente casualizado, sendo os dados submetidos à análise de variância e as médias comparadas pelo teste não paramétrico de KruskalWallis. A comparação entre os tratamentos nos testes com pulverização pré e pós-parasitismo foi feita pelo teste não paramétrico de Mann Whitney, com auxílio do programa estatístico BioEstat 5.0 (AYRES et al., 2007).

\section{Resultados e Discussão}

Teste com chance de escolha não repeliram o parasitismo de $T$. podisi sobre ovos de E. heros, quando comparados às respectivas testemunhas. No entanto, Compostonat, Natualho ${ }^{\circledR}$ e Óleo de Gerânio reduziram o parasitismo com $12,4 \%, 26,3 \%$ e $27,3 \%$ de ovos parasitados, respectivamente (Tabela 2). Essa diferença observada pode estar relacionada à composição do produto, formulação, concentração do ingrediente ativo e ao próprio parasitoide (HOHMANN; SILVA; NOVAES, 2010), pois no processo de parasitismo, para a localização e o reconhecimento do hospedeiro a curtas distâncias estão envolvidos estímulos visuais e olfativos (cairomônios) (VINSON, 1997), enquanto que a aceitação do hospedeiro está condicionada a estímulos visuais e gustativos (FATOUROS et al., 2005). Assim, no caso da análise sensorial, a presença de algumas substâncias, com propriedades não desejáveis na superfície do ovo do hospedeiro, ao serem identificadas pelo parasitoide, pode desencadear a rejeição ao parasitismo.

Os produtos Rotenat ${ }^{\circledR}$, Pironat $^{\circledR}$ e Natuneem ${ }^{\circledR}$

Tabela 2. Taxa de parasitismo ( \pm EP) de Telenomus podisi em ovos de Euschistus heros, pulverizados com produtos alternativos, em bioensaios com chance de escolha. Temperatura de $26 \pm 2^{\circ} \mathrm{C}, 12$ horas de fotofase e UR de $75 \pm 10 \%$.

\begin{tabular}{cc}
\hline Tratamento & Taxa de parasitismo (\%) $^{\mathbf{1}}$ \\
\hline Testemunha & $87,6 \pm 5,18 \mathrm{a}$ \\
Compostonat & $12,4 \pm 5,18 \mathrm{~b}$ \\
\hline$P$ & 0,01 \\
\hline Testemunha $^{\circledR}$ & $62,2 \pm 8,61 \mathrm{a}$ \\
Rotenat $^{\circledR}$ & $37,8 \pm 8,61 \mathrm{a}$ \\
\hline$P$ & 0,28 \\
\hline Testemunha $^{\text {Natualho }}{ }^{\circledR}$ & $73,7 \pm 7,91 \mathrm{a}$ \\
\hline$P$ & $26,3 \pm 7,91 \mathrm{~b}$ \\
\hline Testemunha $^{\circledR}$ & 0,02 \\
\hline Pironat $^{\circledR}$ & $46,2 \pm 8,59 \mathrm{a}$ \\
\hline$P$ & $53,8 \pm 8,59 \mathrm{a}$ \\
\hline Testemunha $^{\text {Natuneem }}{ }^{\circledR}$ & 0,55 \\
\hline$P$ & $67,6 \pm 8,83 \mathrm{a}$ \\
\hline Testemunha & $32,4 \pm 8,83 \mathrm{a}$ \\
\hline Óleo de Gerânio & 0,10 \\
\hline$P$ & $72,7 \pm 8,66 \mathrm{a}$ \\
\hline
\end{tabular}

${ }^{1}$ Médias ( \pm EP) seguidas pela mesma letra não diferem entre si pelo teste de Wilcoxon $(\mathrm{p}<0,05)$.

Fonte: Elaboração dos autores. 
Em estudo com outros himenópteros, Tetragonisca angustula Latreille (Hymenoptera: Apidae) e Nannotrigona testaceicornis Lepitier (Hymenoptera: Apidae), a rotenona, princípio ativo de Rotenat ${ }^{\circledR}$, não provocou repelência a esses insetos, não afetando a distância percorrida, o tempo em movimento e a velocidade de movimentação destes insetos adultos (XAVIER, 2009). Com relação ao produto Pironat ${ }^{\circledR}$, que de acordo com o fabricante, é recomendado como repelente de insetos, no presente trabalho, tal efeito não foi observado.

Natuneem $^{\circledR}$ tem como princípio ativo a azadirachtina, que, de acordo com Mossini e Kemmelmeier (2005), é um terpenoide amplamente conhecido e eficiente no controle de pragas, agindo como repelente, fagodeterrente e regulador de crescimento. Entretanto, no presente estudo, esse produto não causou repelência ao parasitismo de $T$. podisi. Por outro lado, em estudo com extrato aquoso de sementes de nim $(10 \%)$ e o produto Natural Neem, ambos com o princípio ativo azadirachtina, verificaram-se efeito repelente a $T$. pretiosum (GONÇALVES-GERVÁSIO; VENDRAMIM, 2004; SILVA, 2010). Tal variação nos resultados pode estar relacionada a vários fatores, dentre eles à forma de obtenção dos compostos, formulação e concentração do princípio ativo avaliados.

A repelência observada por Compostonat provavelmente deve-se a sua composição, que é uma combinação de óleos essenciais de diferentes espécies vegetais. Dentre os componentes com efeito repelente a parasitoides, destaca-se o nim (GONÇALVES-GERVÁSIO; VENDRAMIM, 2004). Já o produto Natualho ${ }^{\circledR}$, composto de extrato de alho, também provocou repelência, possivelmente pelo odor acentuado, causado pela alicina (MENDES, 2008), que pode ter interferido no reconhecimento do hospedeiro por $T$. podisi, uma vez que é característica deste tipo de parasitoide, segundo Vinson (1997), tatear os ovos do hospedeiro e fazer o caminhamento sobre este para então ovipositar.
O Óleo de Gerânio, que também repeliu o parasitismo de $T$. podisi, é composto de geraniol e citronelol, que dentre outras indicações são repelentes e inseticidas (IAC, 2010). Essas funções foram evidenciadas por Lima et al. (2008), os quais observaram que o composto geraniol provocou repelência ao pulgão-da-couve Brevicoryne brassicae L. (Hemiptera: Aphidae). Em trabalhos sobre os efeitos de citronelol na alimentação de himenópteros, foi verificada a repelência a Apis mellifera L. (Hymenoptera: Apidae) (MALERBOSOUZA; NOGUEIRA-COUTO (1998) e repelência e toxicidade a T. angustula (XAVIER et al., 2010).

\section{Teste sem chance de escolha}

Quando fêmeas de T. podisi foram confinadas com ovos de E. heros previamente pulverizados com os produtos alternativos, constatou-se que as taxas de parasitismo para Natualho ${ }^{\circledR}$ e Óleo de Gerânio foram significativamente menores quando comparadas às respectivas testemunhas o que também foi observado no teste com chance de escolha (Tabela 2). Para os demais produtos não foi verificada alteração na taxa de parasitismo. Salienta-se que para Natuneem ${ }^{\circledR}$, cujo princípio é azadirachtina, os resultados foram semelhantes aos verificados em estudos com $T$. pretiosum com óleo de nim emulsionável em diferentes concentrações (OLIVEIRA; PRATISSOLI; BUENO, 2003), óleo de nim e extrato pirolenhoso, no pré-parasitismo (MORANDI FILHO et al., 2006) e Natural Neem no pré e pós-parasitismo (SILVA, 2010).

$\mathrm{Na}$ pulverização feita pós-parasitismo, Rotenat ${ }^{\circledR}$ foi o único produto que causou redução na porcentagem de ovos de E. heros com sinal de parasitismo por T. podisi (16\%) quando comparado à sua testemunha $(33,2 \%)$ (Tabela 3). Pode-se inferir, neste caso, que o produto possa ter penetrado no ovo do hospedeiro já parasitado, ocasionado a morte de T. podisi, antes que o mesmo chegasse a fase de pré-pupa. A rotenona provoca inibição na cadeia respiratória mitocondrial, reduzindo o consumo 
de oxigênio (IPCS, 1992) e a produção de ATP (adenosina trifosfato), necessários ao metabolismo e desenvolvimento do organismo, fatores que podem ter ocasionado a morte do parasitoide.

Para a emergência de adultos de T. podisi, em pulverização em pré e pós-parasitismo, os produtos Natuneem $^{\circledR}$, Rotenat ${ }^{\circledR}$, Natualho ${ }^{\circledR}$ e Pironat $^{\circledR}$ não diferiram da testemunha. Também não houve diferença significativa quando se comparou a estratégia de pulverização. Entretanto, os produtos Compostonat e Óleo de Gerânio, na pulverização pré-parasitismo, reduziram significativamente a porcentagem de emergência quando comparados às respectivas testemunhas (Tabela 4 ).

$\mathrm{O}$ produto Pironat $^{\circledR}$, pulverizado em préparasitismo, e Natualho $^{\circledR}$, em pós-parasitismo, apresentaram porcentagem de emergência de (100\%), apresentando respectivamente 1,4 e 1,01 indivíduos por ovo (Tabela 4). Quando Pironat ${ }^{\circledR}$ foi pulverizado previamente, a fêmea de T. podisi, percebendo o produto, pode ter colocado mais de um ovo por hospedeiro como estratégia de manutenção à espécie, comportamento descrito para T. pretiosum, por Vinson (1997).
A razão sexual de $T$. podisi, emergidos de ovos de E. heros, pulverizados previamente ao parasitismo não foi afetada, entretanto, para Natualho ${ }^{\circledR}$ e Óleo de Gerânio, no pós-parasitismo, houve um decréscimo na razão sexual para respectivamente 0,4 e 0,3 (Tabela 5). Na pulverização pós-parasitismo dos demais produtos não houve interferência na razão sexual. De acordo com Vinson (1997), a determinação do sexo dos parasitoides ocorre no ato da postura, variando de acordo com as características físico-químicas e nutricionais do ovo hospedeiro, sendo que em condições adversas são colocados ovos que originarão machos, alterando a razão sexual. No entanto, fatores ambientais podem interferir no desenvolvimento dos parasitoides no ovo do hospedeiro, o que, variavelmente, poderá prejudicar um dos sexos. Neste sentido, é provável que a penetração do produto alternativo no ovo do hospedeiro tenha afetado o ciclo biológico de $T$. podisi, matando as fêmeas que são mais exigentes nutricionalmente. Assim, os resultados observados para a razão sexual na pulverização pós-parasitismo referem-se à razão sexual dos emergidos e não a razão sexual que é selecionada pela fêmea de $T$. podisi no ato do parasitismo.

Tabela 3. Taxa de parasitismo ( \pm EP) de Telenomus podisi em ovos de Euschistus heros, pulverizados com produtos alternativos, em bioensaios sem chance de escolha. Temperatura $26 \pm 2{ }^{\circ} \mathrm{C}, 12$ horas de fotofase e U.R. de $75 \pm 10 \%$.

\begin{tabular}{ccc}
\hline & Taxa de parasitismo (\%) & \\
\hline Tratamento & Pré-Parasitismo & Pós-Parasitismo \\
\hline Testemunha & $32,4 \pm 3,32 \mathrm{a}$ & $33,2 \pm 3,93 \mathrm{ab}$ \\
Natuneem $^{\circledR}$ & $38,8 \pm 4,25 \mathrm{a}$ & $29,4 \pm 5,01 \mathrm{bc}$ \\
Rotenat $^{\circledR}$ & $30,2 \pm 6,02 \mathrm{a}$ & $16,0 \pm 2,28 \mathrm{c}$ \\
Compostonat $_{P}$ & $27,8 \pm 4,24 \mathrm{a}$ & $50,8 \pm 4,44 \mathrm{a}$ \\
\hline Testemunha $_{\text {Natualho }}$ & 0,45 & $<0,01$ \\
\hline$P$ & $79,8 \pm 3,11 \mathrm{a}$ & $65,6 \pm 4,70 \mathrm{a}$ \\
\hline Testemunha $^{\circledR}$ & $31,1 \pm 6,22 \mathrm{~b}$ & $66,0 \pm 5,73 \mathrm{a}$ \\
\hline Pironat & $<0,01$ & 0,68 \\
\hline Óleo de Gerânio & $51,4 \pm 3,12 \mathrm{a}$ & $46,8 \pm 5,27 \mathrm{~b}$ \\
\hline$P$ & $39,3 \pm 4,39 \mathrm{ab}$ & $57,6 \pm 2,80 \mathrm{ab}$ \\
\hline
\end{tabular}

${ }^{1}$ Médias ( \pm EP) seguidas pela mesma letra não diferem entre si pelo teste de Kruskal Wallis $(\mathrm{p}<0,05)$.

Fonte: Elaboração dos autores. 
Tabela 4. Porcentagem ( \pm EP) de Telenomus podisi emergidos de ovos de Euschistus heros pulverizados com produtos alternativos em bioensaios sem chance de escolha em laboratório. Temperatura $26 \pm 2^{\circ} \mathrm{C}, 12$ horas de fotofase e U.R. de $75 \pm 10 \%$.

\begin{tabular}{|c|c|c|c|}
\hline \multirow[b]{2}{*}{ Tratamento } & \multicolumn{3}{|c|}{ Porcentagem de Emergência } \\
\hline & Pré-Parasitismo & Pós-Parasitismo & $\boldsymbol{P}$ \\
\hline Testemunha & $79,7 \pm 0,76 \mathrm{a}$ & $71,1 \pm 5,66 \mathrm{a}$ & \\
\hline Natuneem $^{\circledR}$ & $58,7 \pm 14,11 \mathrm{aA}$ & $57,0 \pm 6,77 \mathrm{aA}$ & 0,45 \\
\hline Rotenat $^{\circledR}$ & $70,2 \pm 10,34 \mathrm{aA}$ & $69,9 \pm 13,94 \mathrm{aA}$ & 0,78 \\
\hline Compostonat & $11,0 \pm 3,89 \mathrm{bB}$ & $77,5 \pm 3,94 \mathrm{aA}$ & $<0,01$ \\
\hline$P$ & $<0,01$ & 0,22 & \\
\hline Testemunha & $87,7 \pm 2,81 \mathrm{a}$ & $95,3 \pm 9,81 \mathrm{~b}$ & \\
\hline Natualho $^{\circledR}$ & $91,4 \pm 7,50 \mathrm{aA}$ & $100,0 \pm 12,10 \mathrm{aA}$ & 0,94 \\
\hline$P$ & 0,66 & $<0,01$ & \\
\hline Testemunha & $99,1 \pm 7,16 \mathrm{a}$ & $78,7 \pm 7,16 \mathrm{a}$ & \\
\hline Pironat $^{\circledR}$ & $100,0 \pm 9,13 \mathrm{aA}$ & $88,8 \pm 10,72 \mathrm{aB}$ & $<0,01$ \\
\hline Óleo de Gerânio & $80,3 \pm 16,93 \mathrm{bA}$ & $83,8 \pm 8,32 \mathrm{aA}$ & 0,98 \\
\hline$P$ & $<0,01$ & 0,82 & \\
\hline
\end{tabular}

Médias ( \pm EP) seguidas pela mesma letra, minúscula na coluna, não diferem entre si, pelo teste de Kruskal Wallis ( $<<0,05)$. Médias( \pm EP) seguidas pela mesma letra, maiúscula na linha, não diferem entre si, pelo teste de Mann Whitney $(\mathrm{p}<0,05)$.

Fonte: Elaboração dos autores.

Os resultados observados nas testemunhas neste estudo corroboram com os obtidos por Torres, Pratissoli e Zanuncio (1997), que observaram valores da razão sexual entre 0,8 e 0,9 para $T$. podisi e Pacheco e Corrêa-Ferreira (1998) e Peres e CorrêaFerreira (2004) que obtiveram variação de 0,6 a 0,8 . Isto evidencia que Natualho ${ }^{\circledR}$, Pironat ${ }^{\circledR}$ e Óleo de Gerânio interferiram na razão sexual, resultando em valores abaixo de 0,5 (Tabela 5).

Os valores de razão sexual entre 0,5 e 0,8 são considerados satisfatórios, pois valores maiores ou menores podem prejudicar o controle biológico. Se ocorrer redução na razão sexual, o número de machos passa a ser maior, resultando na redução no número de parasitoides emergidos da próxima geração. Entretanto, valores maiores que 0,8 também são desfavoráveis, pois haverá um número muito maior de fêmeas do que machos, reduzindo a possibilidade de cópula. Além disso, fêmeas partenogenéticas colocam ovos que darão origem a machos, podendo, também, comprometer a geração futura (POTRICH, 2010).

Com relação ao período ovo-adulto, para machos e para fêmeas emergidas de ovos de E. heros, não houve interferência dos produtos Natuneem $^{\circledR}$, Rotenat ${ }^{\circledR}$ e Compostonat, tanto na comparação com suas respectivas testemunhas, quanto na comparação entre as estratégias de pulverização (Tabela 6). Resultados semelhantes foram observados com óleo de nim aplicado em ovos de A. kuehniella no pré e pós-parasitismo de T. pretiosum (SILVA, 2010). 
Tabela 5. Razão sexual ( \pm EP) de Telenomus podisi emergidos de ovos de Euschistus heros pulverizados com produtos alternativos em bioensaios sem chance de escolha em laboratório. Temperatura $26 \pm 2^{\circ} \mathrm{C}, 12$ horas de fotofase e U.R. de $75 \pm 10 \%$.

\begin{tabular}{cccc}
\hline & \multicolumn{2}{c}{ Razão sexual } & \\
\hline Tratamento & Pré-parasitismo & Pós-Parasitismo & \\
\hline Testemunha & $0,6 \pm 0,05 \mathrm{a}$ & $0,8 \pm 0,04 \mathrm{a}$ & 0,68 \\
Natuneem $^{\circledR}$ & $0,7 \pm 0,07 \mathrm{aA}$ & $0,7 \pm 0,06 \mathrm{aA}$ & 0,02 \\
Rotenat $^{\circledR}$ & $0,7 \pm 0,10 \mathrm{aB}$ & $0,8 \pm 0,07 \mathrm{aA}$ & 0,03 \\
Compostonat & $0,5 \pm 0,14 \mathrm{aB}$ & $0,9 \pm 0,02 \mathrm{aA}$ & \\
\hline$P$ & 0,47 & 0,05 & 0,29 \\
\hline Testemunha $_{\text {Natualho }}{ }^{\circledR}$ & $0,7 \pm 0,04 \mathrm{a}$ & $0,8 \pm 0,04 \mathrm{a}$ & \\
\hline$P$ & $0,5 \pm 0,07 \mathrm{aA}$ & $0,4 \pm 0,06 \mathrm{bA}$ & 0,03 \\
\hline Testemunha & 0,51 & $<0,01$ & $<0,01$ \\
\hline Pironat & $0,6 \pm 0,08 \mathrm{a}$ & $0,7 \pm 0,06 \mathrm{a}$ \\
Óleo de Gerânio & $0,4 \pm 0,09 \mathrm{aB}$ & $0,7 \pm 0,06 \mathrm{aA}$ & \\
\hline$P$ & $0,7 \pm 0,06 \mathrm{aA}$ & $0,3 \pm 0,07 \mathrm{bB}$ & $<0,01$ \\
\hline
\end{tabular}

Médias ( \pm EP) seguidas pela mesma letra, minúscula na coluna, não diferem entre si, pelo teste de Kruskal Wallis $(\mathrm{p}<0,05)$. Médias( \pm EP) seguidas pela mesma letra, maiúscula na linha, não diferem entre si, pelo teste de Mann Whitney ( $<<0,05)$. Fonte: Elaboração dos autores.

Tabela 6. Período ovo-adulto ( \pm EP) de fêmeas e machos de Telenomus podisi emergidos de ovos de Euschistus heros pulverizados com produtos alternativos em bioensaios sem chance de escolha em laboratório. Temperatura $26 \pm 2^{\circ} \mathrm{C}$, 12 horas de fotofase e U.R. de $75 \pm 10 \%$.

\begin{tabular}{|c|c|c|c|c|c|c|}
\hline \multirow[t]{2}{*}{ Tratamento } & \multicolumn{3}{|c|}{ Período ovo-adulto fêmeas } & \multicolumn{3}{|c|}{ Período ovo-adulto machos } \\
\hline & $\begin{array}{c}\text { Pré- } \\
\text { parasitismo }\end{array}$ & $\begin{array}{c}\text { Pós- } \\
\text { parasitismo }\end{array}$ & $\mathbf{p}$ & $\begin{array}{c}\text { Pré- } \\
\text { parasitismo }\end{array}$ & $\begin{array}{c}\text { Pós- } \\
\text { parasitismo }\end{array}$ & $\mathbf{p}$ \\
\hline Testemunha & $13,7 \pm 0,22 \mathrm{a}$ & $13,3 \pm 0,12 \mathrm{a}$ & & $12,8 \pm 0,17 \mathrm{a}$ & $12,5 \pm 0,15 \mathrm{a}$ & \\
\hline Natuneem $^{\circledR}$ & $13,3 \pm 0,25 \mathrm{aA}$ & $13,3 \pm 0,18 \mathrm{aA}$ & 0,84 & $13,0 \pm 0,04 \mathrm{aA}$ & $13,5 \pm 0,47 \mathrm{aA}$ & 0,19 \\
\hline Rotenat $^{\circledR}$ & $13,3 \pm 0,17 \mathrm{aA}$ & $13,0 \pm 0,21 \mathrm{aA}$ & 0,14 & $12,6 \pm 0,49 \mathrm{aA}$ & $12,5 \pm 0,34 \mathrm{aA}$ & 0,26 \\
\hline Compostonat & $13,2 \pm 0,40 \mathrm{aA}$ & $13,3 \pm 0,14 \mathrm{aA}$ & 0,47 & $12,0 \pm 0,27 \mathrm{aA}$ & $12,7 \pm 0,49 \mathrm{aA}$ & 0,25 \\
\hline $\mathrm{p}$ & 0,64 & 0,05 & & 0,09 & 0,06 & \\
\hline Testemunha & $13,2 \pm 0,11 \mathrm{a}$ & $13,0 \pm 0,10 \mathrm{a}$ & & $11,6 \pm 0,09 \mathrm{~b}$ & $11,3 \pm 0,09 b$ & \\
\hline Natualho $^{\circledR}$ & $11,2 \pm 0,35 \mathrm{bA}$ & $13,7 \pm 0,44 \mathrm{aA}$ & $<0,05$ & $13,0 \pm 0,17 \mathrm{aA}$ & $13,5 \pm 0,34 \mathrm{aA}$ & 0,63 \\
\hline $\mathrm{p}$ & $<0,01$ & 0,05 & & $<0,01$ & $<0,01$ & \\
\hline Testemunha & $16,8 \pm 0,14 \mathrm{a}$ & $16,8 \pm 0,16 \mathrm{a}$ & & $16,4 \pm 0,11 \mathrm{a}$ & $16,0 \pm 0,10 \mathrm{a}$ & \\
\hline Pironat ${ }^{\circledR}$ & $17,0 \pm 0,19 \mathrm{aA}$ & $16,9 \pm 0,08 \mathrm{aA}$ & 0,96 & $16,1 \pm 0,05 \mathrm{aA}$ & $15,8 \pm 0,12 \mathrm{aB}$ & 0,01 \\
\hline Óleo de gerânio & $17,1 \pm 0,09 \mathrm{aA}$ & $16,7 \pm 0,14 \mathrm{aA}$ & 0,07 & $16,2 \pm 0,14 \mathrm{aA}$ & $16,2 \pm 0,07 \mathrm{bA}$ & 0,62 \\
\hline $\mathrm{p}$ & 0,45 & 0,50 & & 0,31 & 0,03 & \\
\hline
\end{tabular}

Médias ( \pm EP) seguidas pela mesma letra, minúscula na coluna, não diferem entre si, pelo teste de Kruskal Wallis ( $p<0,05)$. Médias( \pm EP) seguidas pela mesma letra, maiúscula na linha, não diferem entre si, pelo teste de Mann Whitney ( $\mathrm{p}<0,05)$.

Fonte: Elaboração dos autores. 
$\mathrm{Na}$ pulverização pré-parasitismo, Natualho ${ }^{\circledR}$ provocou redução significativa no período ovoadulto de fêmeas de $T$. podisi para 11,2 dias, enquanto na testemunha foi de 13,2 dias. Já para machos, tanto na pulverização em pré, quanto em pós-parasitismo o período ovo-adulto foi mais longo, com 13,0 e 13,5, respectivamente, comparados às suas testemunhas. Isto pode ser considerado um fator negativo para o controle em campo, pois acarretaria na demora de emergência de adultos, resultando na diminuição do número da progênie ao longo do tempo (POTRICH, 2010). No entanto, é importante salientar que embora tenha ocorrido essa variação significativa, os resultados demonstram alteração de apenas algumas horas no período, o que provavelmente não comprometeria a manutenção da população em campo. Tal diferença pode estar relacionada ao tempo de seleção do ovo para o parasitismo em função da presença dos produtos.

Essa variação no período ovo-adulto pode estar relacionada ao comprometimento da qualidade do hospedeiro com a aplicação dos referidos produtos alternativos, pois, segundo Vinson (1997), o desenvolvimento do parasitoide pode ser acelerado ou retardado, dependendo da qualidade do hospedeiro.

Natuneem $^{\circledR}$ foi o único produto alternativo considerado seletivo ao parasitoide de ovos $T$. podisi, por não interferir nas variáveis avaliadas, enquanto os demais produtos avaliados interferiram negativamente em um ou mais parâmetros biológicos.

\section{Referências}

AGROFIT. Sistema de agrotóxicos fitossanitários. Brasília: Ministério da Agricultura, 2012. Disponível em: $\quad<$ http://extranet.agricultura.gov.br/agrofit_cons/ principal_agrofit_cons> Acesso em: 10 maio 2011.

AYRES, M.; AYRES, J. R.; AYRES, D. L.; SANTOS, A. S. BioEstat 5.0 - aplicações nas áreas de ciências biológicas e médicas. Belém: Sociedade Civil Mamirauá, 2007. $364 \mathrm{p}$.
BELORTE, L. C.; RAMIRO, Z. A.; FARIA, A. M.; MARINO, C. A. B. Danos causados por percevejos (Hemiptera: Pentatomidae) em cinco cultivares de soja (Glycine $\max ($ L.) Merrill, 1917) no município de Araçatuba, SP. Arquivos do Instituto Biológico, São Paulo, v. 70, n. 2, p. 169-175, 2003.

BROGLIO-MICHELETTI, S. M. F.; SANTOS, A. J. N.; PEREIRA-BARROS, J. L. Ação de alguns produtos fitossanitários para adultos de Trichogramma galloi Zucchi, 1988 (Hymenoptera: Trichogrammatidae). Ciência e Agrotecnologia, Lavras, v. 30, n. 6, p. 10511055, 2006.

CORRÊA-FERREIRA, B. S.; MOSCARDI, F. Seasonal occurrence and host spectrum of egg parasitoids associated with soybean stink bugs. Biological Control, Orlando, v. 5, n. 2, p. 196-202, 1995.

CORRÊA-FERREIRA， B. S.; PERES, W. A. A. Alternativas para o manejo dos percevejos-pragas (Hemiptera: Pentatomidae) em sistema de soja orgânica. Revista Brasileira de Agroecologia, Cruz Alta, v. 1, n. 1, p. 649-652, 2006.

FATOUROS, N. E.; KISS, B. G.; KALKERS, L. A.; GABORENA, R. S.; DICKE, M.; HILJER, M. Oviposition induced plants cues: do they arrest Trichogramma wasps during host location? Entomologia Experimentalis et Applicata, Holanda, v. 115, n. 1, p. 207-215, 2005.

GODOY, K. B.; ÁVILA, C. J.; DUARTE, M. M.; ARCE, C. C. M. Parasitismo e sítios de diapausa de adultos do percevejo marrom, Euschistus heros na região da Grande Dourados, MS. Ciência Rural, Santa Maria, v. 40, n. 5, p. 1199-1202, 2010.

GONÇALVES-GERVÁSIO, R. C. R.; VENDRAMIM, J. D. Efeito de extratos de Meliáceas sobre o parasitóide de ovos Trichograma pretiosum Riley (Hymenoptera: Trichogrammatidae). Neotropical Entomology, Londrina, v. 33, n. 5, p. 607-612, 2004.

HOHMANN, F.; SILVA, F. A. C.; NOVAES, T. G. de. Selectivity of Neem to Trichogramma pretiosum Riley and Trichogramma annulata De Santis (Hymenoptera: Trichogrammatidae). Neotropical Entomology, Londrina, v. 39, n. 6, p. 985-990, 2010.

INSTITUTO AGRONÔMICO DE CAMPINAS - IAC. Gerânio (Pelargonium sp.). 2010. Disponível em: $<$ http:// iac.sp.gov.br/Tecnologias/Geranio/geranio.htm>. Acesso em: 08 dez. 2010.

INTERNATIONAL PROGRAMA EM CHEMICAL SAFETY - IPCS. Rotenone health and safety guide. 1992. 17 p. (World Health Organization, n. 73). 
LIMA, R. K.; CARDOSO, M. G.; MORAES, J. C.; VIEIRA, S. S.; MELO, B. A.; FILGUEIRA, C. C. Composição dos óleos essenciais de Anis-estrelado Illicium verum L. e de Capim-limão Cymbopogon citratus (DC.) Stapf: avaliação do efeito repelente sobre Brevicoryne brassicae (L.) (Hemiptera: Aphididae). Bioassay, Piracicaba, v. 3, n. 8, p. 1-6, 2008.

MALERBO-SOUZA, D. T.; NOGUEIRA-COUTO, R. H. Efeitos de atrativos e repelentes sobre o comportamento da abelha (Apis mellifera L). Scientia Agricola, Piracicaba, v. 55, n. 3, p. 388-394, 1998.

MEDEIROS, L.; MEIGER, G. Ocorrência e desempenho de Euschistus heros (F.) (Heteroptera: Pentatomidae) em plantas hospedeiras alternativas no Rio Grande do Sul. Neotropical Entomology, Londrina, v. 38, n. 4, p. 459463, 2009.

MENDES, P. A. P. Estudo do teor de alicina em alho. 2008. Dissertação (Mestrado em Engenharia Química) Escola Superior de Tecnologia e de Gestão, Bragança.

MORANDI FILHO, W. J.; BOTTON, M.; GRÜTZMACHER, A. D.; GIOLO, F. P.; MANZONI, C. G. Ação de produtos naturais sobre a sobrevivência de Argyrotaenia sphaleropa (Meyrick) (Lepidoptera: Tortricidae) e seletividade de inseticidas utilizados na produção orgânica de videira sobre Trichogramma pretiosum Riley (Hymenoptera: Trichogrammatidae). Ciência Rural, Santa Maria, v. 36, n. 4, p. 1072-1078, jul./ago, 2006.

MOSSINI, S. A. G.; KEMMELMEIER, C. A árvore Nim (Azadirachta indica A. Juss): múltiplos usos. Acta Farm. Bonaerense, v. 24, n. 1, p. 139-48, 2005.

NUNES, M. C.; CORRÊA-FERREIRA, B. S. Danos causados à soja por adultos de Euschistus heros (Fabricius) (Hemiptera:Pentatomidae), sadios e parasitados por Hexacladia smithii Ashmead (Hymenoptera: Encyrtidae). Neotropical Entomology, Londrina, v. 31, n. 1, p. 109113, 2002.

OLIVEIRA, R. C.; PRATISSOLI, D.; BUENO, A. F. Efeito de Azadiracta indica (nim) sobre o parasitismo de Trichogramma pretiosum (Hymenoptera: Trichogrammatidae) em ovos de Anagasta kuehniella (Lepidoptera: Pyralidae). Revista Ecossistema, São Paulo, v. 28, n. 1-2, p. 75-78, jan./dez. 2003.

PACHECO, D. J. P.; CORRÊA-FERREIRA, B. S. Potencial reprodutivo e longevidade do parasitóide Telenomus podisi Ashmead, em ovos de diferentes espécies de percevejos. Anais da Sociedade Entomológica do Brasil, Londrina, v. 27, n. 4, p. 585-591, 1998.
Parasitismo de Telenomus podisi Ashmead (Hymenoptera: Scelionidae) em populações de percevejos pragas da soja. Anais da Sociedade Entomológica do Brasil, Londrina, v. 29, n. 2, p. 295-302, 2000.

PERES, W. A. A.; CORRÊA-FERREIRA, B. S. Methodology of mass multiplication of Telenomus podisi Ash. and Trissolcus basalis (Woll.) (Hymenoptera: Scelionidae) on eggs of Euschistus heros (Fab.) (Hemiptera: Pentatomidae). Neotropical Entomology, Londrina, v. 33, n. 4, p. 457-462, 2004.

Potencial do óleo de nim como inseticida vegetal no controle dos percevejos-pragas da soja (Hemiptera: Pentatomidae). Revista Brasileira de Agroecologia, Cruz Alta, v. 1, n. 1, p. 1651-1655, 2006.

POTRICH, M.; ALVES, L. F. A.; HAAS, J.; SILVA, E. R. L. da; DAROS, A.; PIETROWSKI, V.; NEVES, P. M. O. J. Seletividade de Beauveria bassiana e Metarhizium anisopliae a Trichograma pretiosum Riley (Hymenoptera: Trichogrammatidae). Neotropical Entomology, Londrina, v. 38, n. 6, p. 822-826, 2009.

POTRICH, M. Seletividade de fungos entomopatogênicos a Trichogramma pretiosum Riley (Hymenoptera: Trichogrammatidae) e virulência a Bemisia tabaci (Hemiptera: Aleyrodidae). 2010. Tese (Doutorado em Agronomia) - Universidade Estadual de Londrina, Londrina.

SCHAEFER, W. C.; PANIZZI, A. R. Heteroptera of Economic importance. Boca Raton, FL: CRC Press LLC, 2000.824 p.

SILVA, E. R. L. da. Efeito de produtos alternativos sobre Bacillus thuringiensis subesp. kurstaki e Trichogramma pretiosum Riley (Hymenoptera: Trichogrammatidae). 2010. Tese (Doutorado em Agronomia) - Universidade Estadual de Londrina, Londrina.

TORRES, J. B.; PRATISSOLI, D.; ZANUNCIO, J. Exigências térmicas e potencial de desenvolvimento dos parasitóides Telenomus podisi Ashmead e Trissolcus brochymenae (Ashmead) em ovos de percevejo predador Podisus nigrispinus (Dallas). Anais da Sociedade Entomológica do Brasil, Londrina, v. 26, n. 3, p. 445453, 1997.

VINSON, S. B. Comportamento de seleção hospedeira de parasitóides de ovos, com ênfase na família Trichogrammatidae. In: PARRA, J. R. P.; ZUCCHI, R. A. (Ed.). Trichogramma e o controle biológico aplicado. Piracicaba: FEALQ, 1997. p. 67-119. 
XAVIER, V. M. Impacto de Inseticidas Botânicos sobre Apis mellifera, Nannotrigona testaceicornis e Tetragonisca angustulata (Hymenoptera: Apidae). 2009. Dissertação (Mestrado em Entomologia) - Universidade Federal de Viçosa, Viçosa.
XAVIER, V. M.; MESSAGE, D.; PICANÇO, M. C.; BACCI, L.; SILVA, G. A.; BENEVENUTE, J. S. Impacto

f Botanical Insecticides on Indigenous Stingless Bees (Hymenoptera: Apidae). Sociobiology, California, v. 56, n. 3, p. 1-13, 2010. 